\title{
Effectiveness of oseltamivir treatment on clinical failure in hospitalized patients with lower respiratory tract infection
}

Timothy L. Wiemken ${ }^{1,4^{*}}$, Stephen P. Furmanek ${ }^{2}$, Ruth M. Carrico ${ }^{2}$, Paula Peyrani ${ }^{2,4}$, Daniel Hoft ${ }^{1}$, Alicia M. Fry ${ }^{3}$ and Julio A. Ramirez ${ }^{2}$

\begin{abstract}
Background: Influenza is associated with excess morbidity and mortality of individuals each year. Few therapies exist for treatment of influenza infection, and each require initiation as early as possible in the course of infection, making efficacy difficult to estimate in the hospitalized patient with lower respiratory tract infection. Using causal machine learning methods, we re-analyze data from a randomized trial of oseltamivir versus standard of care aimed at reducing clinical failure in hospitalized patients with lower respiratory tract infection during the influenza season.
\end{abstract}

Methods: This was a secondary analysis of the Rapid Empiric Treatment with Oseltamivir Study (RETOS). Conditional average treatment effects (CATE) and 95\% confidence intervals were computed from causal forest including 85 clinical and demographic variables. RETOS was a multicenter, randomized, unblinded, trial of adult patients hospitalized with lower respiratory tract infections in Kentucky from 2009 through 2012. Adult hospitalized patients with lower respiratory tract infection were randomized to standard of care or standard of care plus oseltamivir as early as possible after hospital admission but within $24 \mathrm{~h}$ of enrollment. After randomization, oseltamivir was initiated in the treatment arm per package insert. The primary outcome was clinical failure, a composite measure including failure to reach clinical improvement within 7 days, transfer to intensive care $24 \mathrm{~h}$ after admission, or rehospitalization or death within 30 days.

Results: A total of 691 hospitalized patients with lower respiratory tract infections were included in the study. The only subgroup of patients with a statistically significant CATE was those with laboratory-confirmed influenza infection with a $26 \%$ lower risk of clinical failure when treated with oseltamivir (95\% Cl 3.2-48.0\%).

Conclusions: This study suggests that addition of oseltamivir to standard of care may decrease clinical failure in hospitalized patients with influenza-associated lower respiratory tract infection versus standard of care alone. These results are supportive of current recommendations to initiate antiviral treatment in hospitalized patients with confirmed or suspected influenza as soon as possible after admission.

Trial registration Original trial: Clinical Trials.Gov; Rapid Empiric Treatment With Oseltamivir Study (RETOS) (RETOS); ClinicalTrials.gov Identifier: NCT01248715 https://clinicaltrials.gov/ct2/show/NCT01248715

Keywords: Tamiflu, Flu, Heterogenous treatment effects, Causal forest

*Correspondence: timothy.wiemken@health.slu.edu

${ }^{1}$ Division of Infectious Diseases, Allergy, and Immunology, Department of Internal Medicine, Saint Louis University School of Medicine, 1100 South Grand Blvd \#817, Doisy Research Center, St. Louis, MO 63104, USA Full list of author information is available at the end of the article

\section{Introduction}

Influenza is associated with excess morbidity and mortality of individuals each year in the United States and contributes substantially to the national healthcare 
burden each winter [1]. Neuraminidase inhibitors such as oseltamivir, peramivir, and zanamivir are one of three categories of FDA-approved therapies for influenza illness and reduce duration of infection through prevention of virus exit from infected cells [2]. Because of this mechanism, administration of drug early in the course of infection is most efficacious [3]. We previously conducted a randomized trial to evaluate the impact of oseltamivir on clinical outcomes of hospitalized patients with lower respiratory tract infection associated with influenza [4]. In that study, we found limited, and not statistically significant, efficacy of oseltamivir in reducing clinical failure, a composite measure including failure to reach clinical improvement within 7 days, transfer to intensive care $24 \mathrm{~h}$ after admission, or rehospitalization or death within 30 days, in hospitalized patients with influenzaassociated lower respiratory tract infection [4]. Although the average treatment effect was not significant, it is possible that therapy had clinical benefits in subgroups of patients, or our analytical approach was insufficient for the data obtained.

Since the results of this study were published, there have been many innovations in analytical approaches for these types of data, specifically the field of machine learning [5]. These advancements have improved not only our ability to develop predictive models but also allow for computation of treatment effects. In this area, treatment effect computation is also possible across subgroups of individuals, with fewer limitations of sample size and false discovery rates that plague frequentist statistical approaches [6]. Since the sample size of influenza virus infected patients in our initial randomized trial was relatively small and we were underpowered for our primary endpoint, it is possible that we were unable to appropriately detect subgroups in which oseltamivir therapy was efficacious.

The objective of this post hoc study was to utilize a novel machine learning method, the causal forest [7], to evaluate subgroups of hospitalized patients with lower respiratory tract infection who may have differential therapeutic response to oseltamivir therapy for prevention of clinical failure.

\section{Methods}

\section{Design and patients}

This was a secondary analysis of the Rapid Empiric Treatment with Oseltamivir Study (RETOS) [4]. Briefly, RETOS was a randomized, unblinded, trial of adult patients hospitalized with lower respiratory tract infections in Kentucky from 2009 through 2012. Patients were randomized to group A (standard of care) or group B (standard of care plus oseltamivir) as early as possible after hospital admission but within $24 \mathrm{~h}$ of enrollment.
Both per-protocol and intent-to-treat analyses were performed in the original study since all patients with lower respiratory tract infections were randomized regardless of etiology, though subsets with documented influenza virus infection by reverse transcriptase polymerase chain reaction (rt-PCR) were also analyzed. For the purposes of the present study, all patients in the intent-to-treat analysis (randomized patients with lower respiratory tract infection regardless of documented etiology) were included.

\section{Study variables}

A total of 85 variables were used to investigate potential heterogeneity in average treatment effects between oseltamivir and clinical failure. All variables used in the models are included in Fig. 1 of the results. Variables were selected based on clinical interest, complete data availability in the study database, and potential need for adjustment due to confounding effects in the assessment of treatment effects of oseltamivir on clinical failure.

\section{Study outcomes}

The primary outcome was clinical failure, as defined in our original study: a composite measure including failure to reach clinical improvement within 7 days, transfer to intensive care $24 \mathrm{~h}$ after admission, or rehospitalization or death within 30 days.

\section{Human subjects protection}

The University of Louisville Human Subjects Research Protection Program Office (Protocol 10.0465), the Robley Rex VA Medical Center Institutional Review Board (IRB; Protocol 0068/00325), and each participating hospital reviewed and approved the study prior to any enrollment. The Centers for Disease Control and Prevention (CDC) IRB granted reliance on local ethical review approvals as the funding agency of the original study.

\section{Statistical analysis}

Baseline characteristics of patients were compared using Chi-Squared or Fishers Exact tests. Q values were also computed for all variables versus treatment status to account for the increased false discovery rate due to multiple comparison in this analysis. Causal forests were used to estimate conditional average treatment effects for each variable for those treated and untreated with oseltamivir [6-9]. These heterogenous effects were also accounting for all other variables under study via the random forest approach and can be considered unbiased estimates of the absolute effect of the oseltamivir therapy conditioned on membership in a particular subgroup. Causal forests are extensions of the random forest, which split data repeatedly to create decision trees for classification 


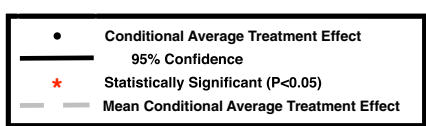

Home wound care me infusion therapy Influenza positive Nursing Home Resident Difficulty using chairs Difficulty bathing Difficulty dressing Right upper lobe infiltrate Tuesday admission Alcoholism
Difficulty using toilet 2010-2011 Influenza Season Cerebrovascular accident Right lower lobe infiltrates Hospitalized $>1$ day in the prior 90 days Community-acquired pneumonia Age 18-49 years

Age $18-49$ years
Right middle lobe infiltrates Left lower lobe infiltrates Difficulty eating Friday admission

Macrolide empiric therapy
Age $50-64$ years Age 50-64 years
Multilobar infiltrates Multilobar infiltrates
Acute bronchitis Fever on admission Current smoker IV antibiotics in the prior 90 days Less than 8 hours to antimicrobial therapy Antibiotic therapy in prior month Long-term systemic steroids Sunday admission Thursday admission Less than 3 days of respiratory symptoms Age $>74$ years
Difficulty walking 2011-2012 Influenza Season Cough on admission Diabetes mellitus
Diabs on admission Interstitial bilateral infiltrates Chronic renal failure Male gender Male gender Active cancer Pulmonary infiltrates (unspecified location) White blood cell count abnormal on admission IV steroids on admission Need for intensive care on admission Congestive heart failure

Saturday admission vaccine for current year Wednesday admission Renal disease
2012-2013 Influenza Season 2012-2013 Influenza Season Age 65-74 years Arterial blood gas obtained Unable to use toilet
Statin use Statin use
Monday admission Monday admission
Unable to bathe chronic bronchitis Unable to walk Unable to use chairs Neurologic disease Home oxygen therapy Admission for CAP in prior year Unable to eat Unable to dress Slood pressure support on admission
Suspicion of aspiration
Pulmonary edema on admission Suspicion of aspiration
Pulmonary edema on admission Serious arrhythmia on admission Mental status changes Mental status changes Acute myocardial infarction on admission
Worsening of long-term arrhythmia on admission.

\section{Conditional Average Treatment Effect (Percent)}

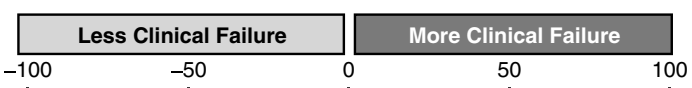

$1+1$

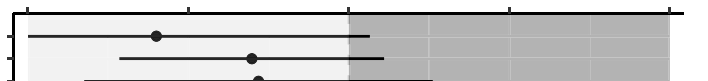

$*$
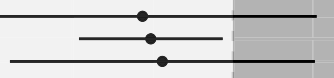

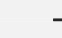

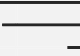

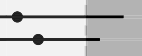

$\because-$

.
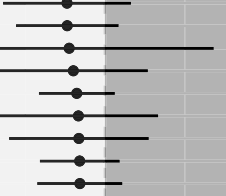

$\because$

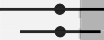

$\longrightarrow$

.

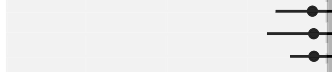

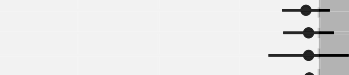

-

Fig. 1 Conditional average treatment effects for clinical failure. Variable listed is the subgroup of patients for which the treatment effect was computed for patients treated versus not treated with oseltamivir 
or regression. The causal forest is built similarly, though instead of assessing the variable selection (splitting rules) based on prediction error, it maximizes the difference in treatment effects for the treatment/outcome pair. For the causal forest, a total of 50,000 trees were used to allow for accurate computation of $95 \%$ confidence intervals. Conditional average treatment effects were extracted for each categorical variable under consideration along with the 95\% confidence interval and a data visualization was created for each outcome. R v4.04 (R Foundation for Statistical Computing, Vienna, Austria) was used for all analyses. The package grf was used for computation of causal forests and extraction of conditional average treatment effects [10].

\section{Results}

A total of 691 hospitalized patients with lower respiratory tract infections randomized to either standard of care or standard of care plus oseltamivir with complete data for the variables under consideration were included in the study. In bivariable analyses (Table 1), two variables (Age category $\geq 75$ years and Wednesday admission to the hospital) were significantly different across treatment status levels based on p-values, though q-values for all variables were 1 , indicating statistically significant p-values were likely due to false discovery as opposed to true differences. Figure 1 depicts the conditional average treatment effect estimates for each variable under study for those treated with oseltamivir versus those who were not treated. For the primary outcome of clinical failure, patients who were documented influenza positive were the only subgroup identified with significant oseltamivir impact. The results identified a $26 \%$ lower occurrence of clinical failure for patients with influenza treated with oseltamivir versus those not treated with oseltamivir (95\% CI 3.2-48.0\%).

\section{Discussion}

This study suggests that addition of oseltamivir to standard of care may decrease clinical failure in hospitalized patients with influenza-associated lower respiratory tract infection versus standard of care alone. These results are reasonably consistent with the reductions in clinical failure with oseltamivir treatment in the per-protocol analysis from our original randomized trial which identified a non-significant reduction from $24 \%$ in the standard of care arm to $14 \%$ in the standard of care plus oseltamivir $\operatorname{arm}(\mathrm{p}=0.414)[4]$.

Causal inference in machine learning is a relatively new addition to the methodologic arsenal [5]. The causal forest, a special version of the generalized random forest [7], is the most widely used method for computing conditional average treatment effects in the medical literature; being used for readmission risk [11], targeted intervention development [6], diabetes epidemiology [12], and identification of risk factors for thyroid disease [13]. As discussed previously, causal machine learning methods are distinct from traditional supervised machine learning since these models estimate the treatment effect as opposed to risk or prediction. Further, they are not bound by parametric assumptions common in traditional methods such as regression modeling and are less apt to overfit through application of regularization in the computation [8, 11]. These factors, along with the ability to assess conditional average treatment effects in small sample sizes allowed us to perform a more robust analysis of these randomized data.

Here, the presence of influenza was the primary driver of significant decreases in clinical failure with oseltamivir therapy. This suggests that our approach to estimate conditional average treatment effects through causal machine learning methods is likely accurate and potentially more useful for detection of subgroup treatment effects in small samples. Future randomized trials may benefit from using similar methodologies as an adjunctive measure for the more traditional frequentist statistical methodologies typically utilized and reported. Use of these novel methods may assist in detection of subgroups where interventions are beneficial or detrimental, allowing for a movement toward more personalized medicine.

This study has several limitations. First, given the small sample size in the study, the variability in our treatment effect estimates is wide, as indicated by many of the $95 \%$ confidence intervals for many variables. Further, we were not able to assess model performance through training and testing given the small sample size, resulting in potentially biased results. Second, the generalizability of treatment effect estimates from causal forest methodologies has yet to be widely documented. These machine learning models for computation of heterogenous treatment effects have only begun to be utilized in medicine $[6,13,14]$ and we were unable to find any studies using these methods in the field of respiratory infections. This study also used a composite outcome, combining several clinical outcomes: failure to reach clinical improvement within 7 days, transfer to intensive care $24 \mathrm{~h}$ after admission, or rehospitalization or death within 30 days. Because of this, we are not able to dissect which individual outcome is impacted by oseltamivir therapy. Further, given this is a novel computational approach for re-analysis of a single randomized clinical trial, we are unable to provide further clinical guidance on use of oseltamivir in the hospitalized patient and suggest continued application of national, regional, and local guidance on antiinfluenza therapy. 
Table 1 Bivariable analysis comparing each of the selected subgroups to study arm

\begin{tabular}{|c|c|c|c|c|}
\hline Demographics & $\begin{array}{l}\text { Treated with } \\
\text { oseltamivir } \\
n=343 \\
n(\%)\end{array}$ & $\begin{array}{l}\text { Not treated with } \\
\text { oseltamivir } \\
n=348 \\
n(\%)\end{array}$ & $p$-value & q-value \\
\hline Age group 18-49 years & $57(16.6)$ & $67(19.3)$ & 0.422 & 1.000 \\
\hline Age group 18-49 years & $136(39.7)$ & $116(33.3)$ & 0.100 & 1.000 \\
\hline Age group 65-74 years & $84(24.5)$ & $70(20.1)$ & 0.197 & 1.000 \\
\hline Age group $\geq 75$ years & $66(19.2)$ & $95(27.3)$ & 0.016 & 1.000 \\
\hline Male gender & $202(58.9)$ & $196(56.3)$ & 0.305 & 1.000 \\
\hline Nursing home resident & $17(5.0)$ & $19(5.5)$ & 0.899 & 1.000 \\
\hline \multicolumn{5}{|l|}{ Signs and symptoms } \\
\hline Cough & $330(96.2)$ & $325(93.4)$ & 0.135 & 1.000 \\
\hline Fever & $145(42.3)$ & $146(42.0)$ & 0.994 & 1.000 \\
\hline Elevated white blood cell count & $227(66.2)$ & $231(66.4)$ & 1.000 & 1.000 \\
\hline \multicolumn{5}{|l|}{ Past medical and social history } \\
\hline Obese $\left(\mathrm{BMI} \geq 30 \mathrm{~kg} / \mathrm{m}^{2}\right)$ & $134(39.1)$ & $136(39.1)$ & 1.000 & 1.000 \\
\hline Active cancer & $46(13.4)$ & $56(16.1)$ & 0.376 & 1.000 \\
\hline Congestive heart failure & $88(25.7)$ & $102(29.3)$ & 0.322 & 1.000 \\
\hline Cerebrovascular accident & $43(12.5)$ & $35(10.1)$ & 0.363 & 1.000 \\
\hline Renal disease & $63(18.4)$ & $62(17.8)$ & 0.929 & 1.000 \\
\hline Liver disease & $23(6.7)$ & $27(7.8)$ & 0.698 & 1.000 \\
\hline Chronic renal failure & $51(14.9)$ & $48(13.8)$ & 0.768 & 1.000 \\
\hline Neurologic disease & $83(24.2)$ & $78(22.4)$ & 0.642 & 1.000 \\
\hline Diabetes mellitus & $120(35.0)$ & $131(37.6)$ & 0.517 & 1.000 \\
\hline History of community acquired pneumonia & $62(18.1)$ & $57(16.4)$ & 0.624 & 1.000 \\
\hline Suspicion of aspiration & $14(4.1)$ & $6(1.7)$ & 0.105 & 1.000 \\
\hline Cirrhosis & $4(1.2)$ & $5(1.4)$ & 1.000 & 1.000 \\
\hline Alcoholism & $8(2.3)$ & $9(2.6)$ & 1.000 & 1.000 \\
\hline History of COPD & $200(58.3)$ & $210(60.3)$ & 0.640 & 1.000 \\
\hline Hospitalized $\geq 2$ days in the prior 90 days & $99(28.9)$ & $114(32.8)$ & 1.000 & 1.000 \\
\hline IV antibiotics in the past 90 days & $88(25.7)$ & $89(25.6)$ & 0.311 & 1.000 \\
\hline Home infusion therapy & $11(3.2)$ & $6(1.7)$ & 0.807 & 1.000 \\
\hline Home wound care & $13(3.8)$ & $11(3.2)$ & 0.562 & 1.000 \\
\hline HIV disease & $10(2.9)$ & $6(1.7)$ & 0.431 & 1.000 \\
\hline Statin use & $133(38.8)$ & $145(41.7)$ & 0.486 & 1.000 \\
\hline Gastroesophogeal reflux diseases & $40(11.7)$ & $53(15.2)$ & 0.207 & 1.000 \\
\hline Pulmonary edema due to congestive heart failure & $3(0.9)$ & $10(2.9)$ & 0.098 & 1.000 \\
\hline Acute myocardial infarction on admission & $8(2.3)$ & $6(1.7)$ & 0.766 & 1.000 \\
\hline Acute worsening of long-term arrhythmia on admission & $8(2.3)$ & $5(1.4)$ & 0.558 & 1.000 \\
\hline Serious arrhythmia on admission & $6(1.7)$ & $15(4.3)$ & 0.082 & 1.000 \\
\hline Antibiotic use in the prior 30 days & $112(32.7)$ & $106(30.5)$ & 0.590 & 1.000 \\
\hline Home oxygen therapy & $76(22.2)$ & $85(24.4)$ & 0.538 & 1.000 \\
\hline Frail & $127(37.0)$ & $136(39.1)$ & 0.633 & 1.000 \\
\hline Unable to bathe & $35(10.2)$ & $42(12.1)$ & 0.511 & 1.000 \\
\hline Unable to dress self & $24(7.0)$ & $23(6.6)$ & 0.959 & 1.000 \\
\hline Unable to walk & $40(11.7)$ & $43(12.4)$ & 0.870 & 1.000 \\
\hline Unable to get in and out of a chair & $18(5.2)$ & $24(6.9)$ & 0.455 & 1.000 \\
\hline Unable to eat & $8(2.3)$ & $12(3.4)$ & 0.517 & 1.000 \\
\hline Unable to use a toilet & $18(5.2)$ & $32(9.2)$ & 0.063 & 1.000 \\
\hline Difficulty bathing & $49(14.3)$ & $54(15.5)$ & 0.728 & 1.000 \\
\hline
\end{tabular}


Table 1 (continued)

\begin{tabular}{|c|c|c|c|c|}
\hline Demographics & $\begin{array}{l}\text { Treated with } \\
\text { oseltamivir } \\
\mathrm{n}=343 \\
\mathrm{n}(\%)\end{array}$ & $\begin{array}{l}\text { Not treated with } \\
\text { oseltamivir } \\
n=348 \\
n(\%)\end{array}$ & p-value & q-value \\
\hline Difficulty dressing self & $44(12.8)$ & $44(12.6)$ & 1.000 & 1.000 \\
\hline Difficulty walking & $75(21.9)$ & $74(21.3)$ & 0.921 & 1.000 \\
\hline Difficulty getting in and out of a chair & $49(14.3)$ & $45(12.9)$ & 0.683 & 1.000 \\
\hline Difficulty eating & $22(6.4)$ & $17(4.9)$ & 0.480 & 1.000 \\
\hline Difficulty using toilet & $43(12.5)$ & $31(8.9)$ & 0.156 & 1.000 \\
\hline Current smoker & $114(33.2)$ & $101(29.0)$ & 0.265 & 1.000 \\
\hline History of influenza vaccine (current season) & $238(69.4)$ & $243(69.8)$ & 0.966 & 1.000 \\
\hline \multicolumn{5}{|l|}{ Type and severity of disease } \\
\hline Community-acquired pneumonia & $236(68.8)$ & $228(65.5)$ & 0.402 & 1.000 \\
\hline Acute exacerbation of COPD & $91(26.5)$ & $102(29.3)$ & 0.466 & 1.000 \\
\hline Acute bronchitis & $16(4.7)$ & $18(5.2)$ & 0.894 & 1.000 \\
\hline Influenza positive & $24(7.0)$ & $33(9.5)$ & 0.294 & 1.000 \\
\hline Arterial blood gas obtained & $157(45.8)$ & $165(47.4)$ & 0.722 & 1.000 \\
\hline Altered mental status on admission & $17(5.0)$ & $15(4.3)$ & 0.824 & 1.000 \\
\hline Need for ventilatory support on admission & $32(9.3)$ & $36(10.3)$ & 0.749 & 1.000 \\
\hline Need for blood pressure support on admission & $15(4.4)$ & $20(5.7)$ & 0.516 & 1.000 \\
\hline Less than 3 days of respiratory symptoms prior to admission & $78(22.7)$ & $95(27.3)$ & 0.195 & 1.000 \\
\hline Need for ICU Care on Admission & $45(13.1)$ & $52(14.9)$ & 0.562 & 1.000 \\
\hline Pneumonia severity index risk class IV or V & $154(44.9)$ & $169(48.6)$ & 0.374 & 1.000 \\
\hline \multicolumn{5}{|l|}{ Radiographic findings } \\
\hline Multilobar infiltrates on chest radiograph & $119(34.7)$ & $114(32.8)$ & 0.647 & 1.000 \\
\hline Pleural effusion & $58(16.9)$ & $73(21.0)$ & 0.205 & \\
\hline Right upper lobe infiltrate on chest radiograph & $32(9.3)$ & $33(9.5)$ & 1.000 & 1.000 \\
\hline Right middle lobe infiltrate on chest radiograph & $40(11.7)$ & $43(12.4)$ & 0.870 & 1.000 \\
\hline Right lower lobe infiltrate on chest radiograph & $108(31.5)$ & $104(29.9)$ & 0.708 & 1.000 \\
\hline Left lower lobe infiltrate on chest radiograph & $95(27.7)$ & $97(27.9)$ & 1.000 & 1.000 \\
\hline Unspecified lobe infiltrate on chest radiograph & $15(4.4)$ & $15(4.3)$ & 1.000 & 1.000 \\
\hline Interstitial infiltrate on chest radiograph & $26(7.6)$ & $18(5.2)$ & 0.254 & 1.000 \\
\hline \multicolumn{5}{|l|}{ Hospitalization and therapeutics } \\
\hline Study year 1 & $81(23.6)$ & $88(25.3)$ & 0.672 & 1.000 \\
\hline Study year 2 & $169(49.3)$ & $172(49.4)$ & 1.000 & 1.000 \\
\hline Study year 3 & $93(27.1)$ & $88(25.3)$ & 0.646 & 1.000 \\
\hline Monday admission to hospital & 49 (14.3) & $50(14.4)$ & 1.000 & 1.000 \\
\hline Tuesday admission to hospital & $60(17.5)$ & $58(16.7)$ & 0.851 & 1.000 \\
\hline Wednesday admission to hospital & $70(20.4)$ & $49(14.1)$ & 0.036 & 1.000 \\
\hline Thursday admission to hospital & $46(13.4)$ & $44(12.6)$ & 0.852 & 1.000 \\
\hline Friday admission to hospital & $47(13.7)$ & $59(17.0)$ & 0.280 & 1.000 \\
\hline Saturday admission to hospital & $36(10.5)$ & $41(11.8)$ & 0.677 & 1.000 \\
\hline Sunday admission to hospital & $35(10.2)$ & $47(13.5)$ & 0.221 & 1.000 \\
\hline$\leq 8 \mathrm{~h}$ from admission to antimicrobial therapy & $269(78.4)$ & $271(77.9)$ & 0.933 & 1.000 \\
\hline Macrolide empiric therapy & $144(42.0)$ & $148(42.5)$ & 0.946 & 1.000 \\
\hline Antithrombotic therapy during hospitalization & $245(71.4)$ & $247(71.0)$ & 0.962 & 1.000 \\
\hline Systemic steroids during hospitalization & $204(59.5)$ & $196(56.3)$ & 0.446 & 1.000 \\
\hline IV steroids on admission & $170(49.6)$ & $169(48.6)$ & 0.852 & 1.000 \\
\hline
\end{tabular}


The strengths of this study include both the data used from the largest randomized trial of hospitalized patients with oseltamivir therapy and the consistency of results from the initial trial using traditional methodologies such as regression modeling.

Future studies may benefit from these methods as adjunctive analytics in randomized trials and potentially for observational designs where appropriate adjustments can be made with collected data. By continuing to perform both methods, we can begin to identify the best analytic approaches to identify more targeted treatments to improve patient outcomes.

In conclusion, this secondary analysis of a randomized clinical trial suggests that oseltamivir may have clinical utility in hospitalized patients with influenza-associated lower respiratory tract infections. These results are supportive of current recommendations to initiate antiviral treatment in hospitalized patients with confirmed or suspected influenza as soon as possible after admission [3].

\section{Acknowledgements}

Not applicable.

\section{Authors' contributions}

TLW: First draft writing, analysis, and interpretation; SPF: analysis and interpretation; RMC, PP, DH: Interpretation; AMF, JAR: Secondary writing and interpretation. All authors read and approved the final manuscript.

\section{Disclaimer}

The findings and conclusions in this report are those of the authors and do not necessarily represent the views of the CDC, US Department of Health and Human Services.

\section{Funding}

This study was not funded. However, the original randomized trial was supported by an award to the University of Louisville by the CDC (Cooperative agreement IP000420-01).

\section{Availability of data and materials}

The datasets generated and/or analyzed during the current study are not publicly available as they contain protected health information but may be available in de-identified format from the corresponding author on reasonable request.

\section{Declarations}

\section{Ethics approval and consent to participate}

The University of Louisville Human Subjects Research Protection Program Office (Protocol 10.0465), the Robley Rex VA Medical Center Institutional Review Board (IRB; Protocol 0068/00325), and each participating hospital reviewed and approved the study prior to any enrollment. The Centers for Disease Control and Prevention (CDC) IRB granted reliance on local ethical review approvals as the funding agency of the original study. All methods were carried out in accordance with the relevant guidelines and regulations (e.g. Helsinki Declaration).

\section{Consent for publication}

Not applicable.

\section{Competing interests}

The authors declare no competing interests.

\section{Author details}

${ }^{1}$ Division of Infectious Diseases, Allergy, and Immunology, Department of Internal Medicine, Saint Louis University School of Medicine, 1100 South Grand Blvd \#817, Doisy Research Center, St. Louis, MO 63104, USA. ${ }^{2}$ Division of Infectious Diseases, Department of Medicine, University of Louisville School of Medicine, Louisville, KY, USA. ${ }^{3}$ Centers for Disease Control and Prevention, Atlanta, Georgia. ${ }^{4}$ Present Address: Pfizer Inc., Collegeville, PA, USA.

Received: 21 June 2021 Accepted: 18 October 2021

Published online: 27 October 2021

\section{References}

1. Centers for Disease Control and Prevention. Disease burden of influenza. https://www.cdc.gov/flu/about/burden/index.html. Updated 5 Oct 2020. Accessed 15 Mar 2021

2. Jackson RJ, Cooper KL, Tappenden P, et al. Oseltamivir, zanamivir and amantadine in the prevention of influenza: a systematic review. J Infect. 2011;62(1):14-25.

3. Uyeki TM, Bernstein HH, Bradley JS, et al. Clinical Practice Guidelines by the Infectious Diseases Society of America: 2018 update on diagnosis, treatment, chemoprophylaxis, and institutional outbreak management of seasonal influenzaa. Clin Infect Dis. 2019;68(6):895-902.

4. Ramirez J, Peyrani P, Wiemken T, Chaves SS, Fry AM. A randomized study evaluating the effectiveness of oseltamivir initiated at the time of hospital admission in adults hospitalized with influenza-associated lower respiratory tract infections. Clin Infect Dis. 2018;67(5):736-42.

5. Wiemken TL, Kelley RR. Machine learning in epidemiology and health outcomes research. Annu Rev Public Health. 2020;41:21-36.

6. Baum A, Scarpa J, Bruzelius E, Tamler R, Basu S, Faghmous J. Targeting weight loss interventions to reduce cardiovascular complications of type 2 diabetes: a machine learning-based post-hoc analysis of heterogeneous treatment effects in the Look AHEAD trial. Lancet Diabetes Endocrinol. 2017;5(10):808-15.

7. Athey S, Tibshirani J, Wager S. Generalized random forests. Ann Stat. 2019;47(2):1148-78.

8. Wager S, Athey S. Estimation and inference of heterogeneous treatment effects using random forests. arXiv:151004342 [math, stat]. 2015.

9. Künzel SR, Sekhon JS, Bickel PJ, Yu B. Metalearners for estimating heterogeneous treatment effects using machine learning. Proc Natl Acad Sci. 2019;116(10):4156-65.

10. Generalized Random Forests [computer program]. Version 1.2.0. 2020.

11. Marafino BJ, Schuler A, Liu VX, Escobar GJ, Baiocchi M. Predicting preventable hospital readmissions with causal machine learning. Health Serv Res. 2020;55(6):993-1002.

12. Elek P, Biro A. Regional differences in diabetes across Europe-regression and causal forest analyses. Econ Hum Biol. 2021;40:100948.

13. Wang L, Cao F, Wang S, Sun M, Dong L. Using k-dependence causal forest to mine the most significant dependency relationships among clinical variables for thyroid disease diagnosis. PLoS ONE. 2017;12(8):e0182070.

14. Scarpa J, Bruzelius E, Doupe P, Le M, Faghmous J, Baum A. Assessment of risk of harm associated with intensive blood pressure management among patients with hypertension who smoke: a secondary analysis of the systolic blood pressure intervention trial. JAMA Netw Open. 2019;2(3):e190005.

\section{Publisher's Note}

Springer Nature remains neutral with regard to jurisdictional claims in published maps and institutional affiliations. 